\title{
Computer graphics and surface perception
}

\author{
ENNIO MINGOLLA \\ Boston University, Boston, Massachusetts
}

\begin{abstract}
Recent advances in computer graphics technology afford the perceptual psychologist studying surface perception new opportunities for parametric control of complex patterns of optical stimulation. These opportunities include the ability to isolate kinds of optical information that are typically confounded in other display media, and the ability to systematically perturb dimensions of optical information that are otherwise difficult, if not impossible, to control. Examples of such methods and of the computer graphics capabilities exploited by the methods are discussed.
\end{abstract}

Gibson's (1950) The Perception of the Visual World inaugurated many contemporary trends in the study of surface perception, but it remained for the recent proliferation of computer graphics technology to bring extensive parametric control to the manipulation of optical information for complex surfaces. Research related to surface perception covers a great many areas, including perception of slant for planar surfaces, of surface curvature, of surface reflectance (gloss or highlights), of transparency, of occlusion or depth-at-an-edge, of cast shadows and illumination conditions, and of rigid versus nonrigid structure. The impression of a surface can come about from numerous sources of information, including texture or luminance gradients, binocular disparity, and various types of motion coherence. Given the multiplicity of issues only hinted at in the preceding litany, our present state of understanding of surface perception reflects an inherent complexity of subject matter. Consider the difficulty in merely attempting to list necessary and sufficient conditions for the perception of a surface.

The point of this paper, however, is not to dwell on the inherent difficulties of the subject matter of surface perception. Quite the contrary, the central thesis is that computer graphics technology is making possible for the first time whole lines of investigation in surface perception. The potential for manipulating vast arrays of pictorial information in systematic and controlled ways does not merely facilitate or improve upon older techniques but rather opens the possibilities for qualitatively new paradigms. Much of the novelty and importance of these techniques lies in their potential for bridging the gap between two long-standing, disparate traditions of perceptual research.

Research in one tradition is characterized by the employment of simple, easily mathematically specified stimuli, such as sine wave gratings. Although such stimuli are not characteristic of "normal" visual input, they have compensating advantages for research. By knowing the

This research was supported in part by the Air Force Office of Scientific Research (AFOSR 85-0149). The author's mailing address is: Center for Adaptive Systems, Boston University, 111 Cummington St., Boston, MA 02215. input precisely, the psychophysicist can measure properties of an unknown mechanism by measuring transformations on a known input, much like a physicist measures (unknown) field properties by passing (known) particles through the field.

A second tradition of perceptual research holds that although the results of research in the first tradition may be accurate for the experimental conditions, the properties of the mechanisms thereby discovered do not generalize to molar perceptual conditions, such as those that exist in our everyday environment, which is filled with illuminated, textured, curved, and moving surfaces. Research in this tradition is often characterized by the use of complex, often ingenious, displays that require a constellation of conditions to produce the perceptual effect. Thus a "demonstration"-a striking "existence proof" or counterexample (e.g., the Ames chair)-is set up. As convincing as these demonstrations can be, they leave many experimentalists baffled by the question of what to do next; that is, how can such demonstrations be perturbed in a way that does not demolish the perceptual effect immediately and in a way that preserves some measure of experimental control for measuring effect magnitudes?

Computer graphics has helped bridge the gap between the desire for rigorous control of experimental variables and the desire to investigate rich optical information for the study of surface perception. Computer graphics techniques provide displays that are richer than those of the more rigorous psychophysical tradition, but that have more parametric manipulability than most of those of the molar tradition. Two aspects of psychophysical methodology for surface perception employing computer graphics rate special mention.

First, with computer graphics, optical information of various kinds can be isolated in a manner that is difficult, if not impossible, using conventional media. Suppose, for example, that a researcher wants to study the impression of shape derived solely from luminance gradients (shading), without confounding textural information. Material surfaces characteristically display both small-scale textural markings due to chemical or physical irregularities and larger scale luminance gradients due to illumination differences and the surface's overall shape. Suppression 
of textural information in the laboratory can be extremely difficult, involving perhaps the careful use of paints and specialized lighting conditions. In a computer graphics simulation of a surface, however, it is a relatively simple matter to ensure that a surface's appearance is modeled only through shading.

The second methodological innovation of note is the potential for using computer graphics to systematically vary entirely new variables of optical stimulation. Instead of being able to control only such variables as luminance or size, the experimenter can manipulate such variables as amount of curvature or exotic textural statistics. Figure 1 shows an example of such a manipulation, in which two objects of different solid shapes are positioned in space so that for each resulting image their projected outer contours occupy precisely the same pixels on a display screen. Thus, the local curvatures of the depicted surfaces vary throughout the areas bounded by the outer object contours, whereas the positions of the outer contours themselves are identical for the two objects. Accordingly, any differences in perceived solid shape can be attributed to internal luminance gradients, without confounding effects from differing outer contours (Mingolla, 1983; Mingolla \& Todd, 1986).

The two advantages just mentioned can be combined with psychophysical methods in unexpected ways. Psychologists have long employed methods that measure the degradation of performance from some standard as a stimulus dimension is varied. Thus, for example, acuity in some task may be related to luminance contrast. Novel analogues of such approaches are being developed for the study of surface perception, because the ability to perturb or distort optical information through computer graphics can reach levels of refinement impossible to achieve by other means. In addition to the capability of producing high-fidelity "realistic" images of complex scenes, the experimenter can use computer graphics to produce systematic transformations of such scenes by isolating and changing certain complex dimensions of stimulation while leaving other dimensions intact. Figure 2 shows an example of such a manipulation. In Figure 2a, textural segments are distributed in a manner appropriate to the constraints of perspective, whereas in Figure $2 b$, the compression of individual textural elements has been suppressed, thereby weakening the impression of solid shape. Such computer-controlled manipulations have been employed by perceptual psychologists for decades (Braunstein, 1976, offers an excellent review of early work in this vein). Most of the early work was done on planar surfaces, however, and recent trends are bringing such methodologies to the study of increasingly complicated surfaces, including curved surfaces and surfaces with self-occluding contours.

The figures included in this article illustrate only a few of the techniques employed recently in research on surface perception. Even so, a description of the techniques (a)

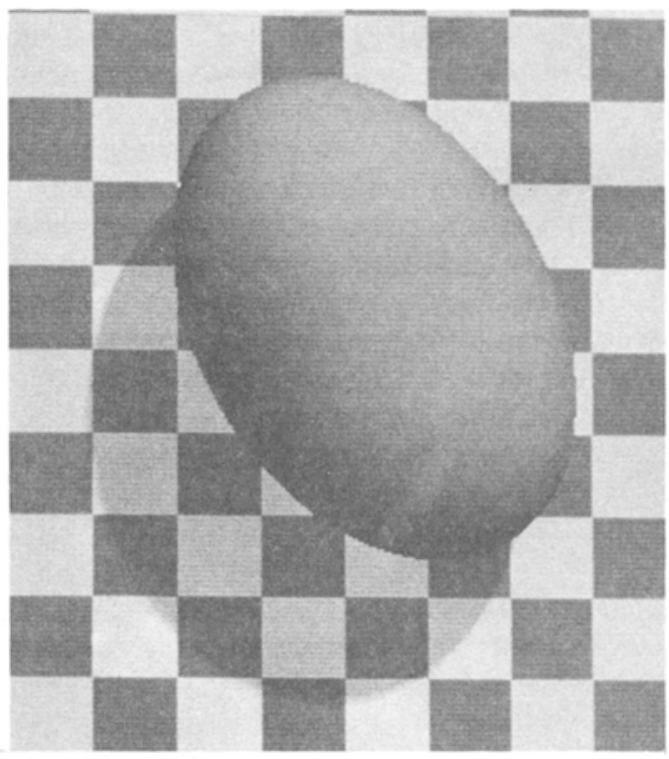

(b)

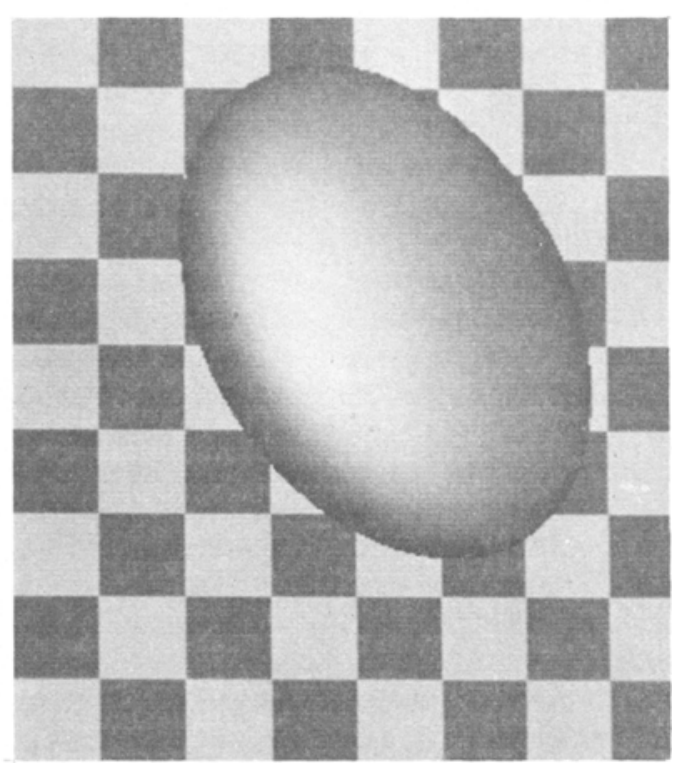

Figure 1. The two ellipsoids depicted in the figure have different computed solid shapes and different appearances. The computed reflectance (shininess or dullness) and illumination directions employed in the pictures vary, and a cast shadow is depicted only in (a). Because the outer contours of the two ellipsoids, relative to the checkerboard background, are identical, no confounding information from outer contour shape can be taken to influence the perceived three-dimensional shapes of the objects. Using displays such as these, Mingolla (1983) and Mingolla and Todd (1980) investigated the contributions of various computed image parameters to perceived shape. From "Perception of solid shape from shading" by E. Mingolla and J. T. Todd, 1986, Biological Cybernetics, 53, 137-151. Reprinted by permission. (It should be noted that because of distortions inherent in photographic reproduction, these images and those in Figure 2 do not look as realistic as they do on a video screen.) 
(a)

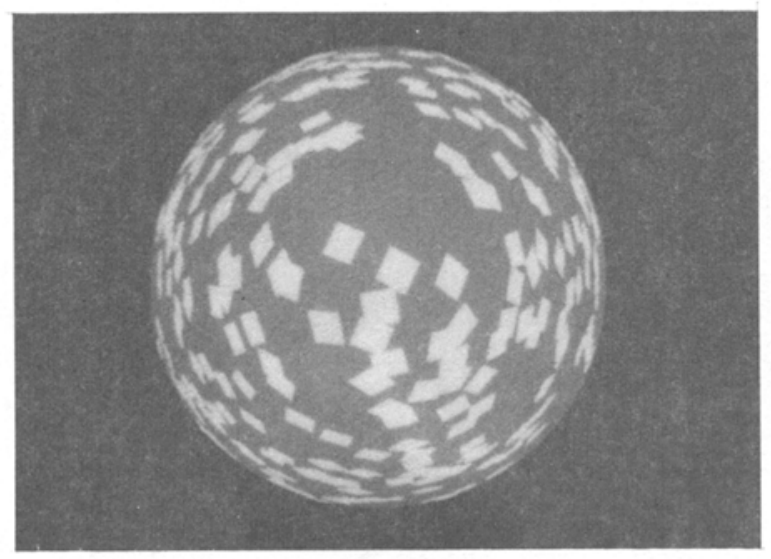

(b)

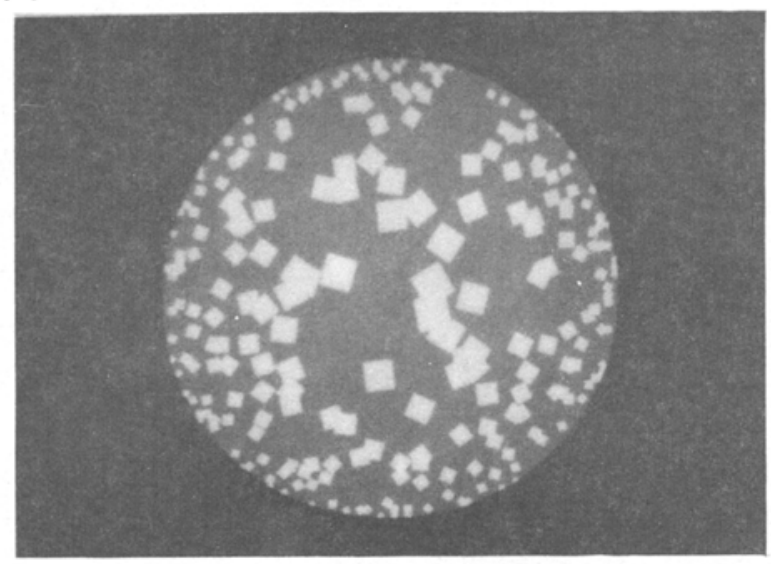

Figure 2. The two textured ellipsoids depicted in this figure are viewed along their longest axes and are computed to have identical solid shapes. The ellipsoid in (a) is rendered according to an accurate model of textural distribution and perspective (Todd \& Mingolla, 1984). For the ellipsoid in (b), however, normal textural compression is suppressed, and only the projected area size of elements is permitted to vary accurately, resulting in a much diminished impression of surface curvature or depth. From "Perception of three dimensional form from patterns of optical texture" by J. T. Todd and R. Akerstrom, in press. Printed by permission.

employed to generate these two figures is beyond the scope of this article. For details regarding Figure 1, readers can consult Mingolla (1983) and Mingolla and Todd $(1984,1986)$; for details on Figure 2, they can refer to Todd and Mingolla (1984) and Todd and Akerstrom (in press). Moreover, for readers with no background in computer graphics, a number of excellent textbooks exist on a range of topics in computer graphics, including those by Foley and van Daam (1984), Newman and Sproull (1979), and Park (1985).

The outlook for the use of computer graphics in surface perception is bright. Apart from the expense of a monitor, the cost per unit of performance of the raster systems needed to produce shaded images continues to decrease rapidly. Two measures of performance in such systems are the number of pixels of resolution and the number of color hues and the intensity available per pixel. These measures covary directly with the availability of digital memory, which continues to get cheaper. Even display processors continue to get faster and cheaper, so that the time needed to generate and display complex images continues to fall. (A display processor is a specialized computer that interprets commands from a host computer and manages the low-level details involved in manipulating what is displayed on a monitor.) Perhaps of even greater significance to researchers is that the ease of use of many computer graphics systems has been improved by the availability of new hardware and software technologies. Three of these will be briefly described: color lookup tables, hardware $z$-buffers, and high-level graphics primitives.

The earliest digital raster display systems used frame buffers without lookup tables. A frame buffer is simply an array of memory registers that maps one-to-one with the pixels on a display screen. For example, consider the digital representation of a gray-level image. If the frame buffer register corresponding to the coordinates $(4,3)$ contains the value 37 , then the pixel in the fourth row and third column of the attached display screen glows with intensity 37 on some scale, perhaps ranging from 0 to 255 for a typical "eight-bit" frame buffer. A color system would require the specification of three such intensities, one each for red, green, and blue channels. The color lookup table introduces a convenient mechanism for specifying how to color any pixel location with a mixture of three intensities. Once again, suppose the register location $(4,3)$ contains the value 37 . On systems with color lookup tables, this 37 would not refer to any intensity as such, but to the address 37 in tables for each of the three primary colors. In location Red-37 might be the intensity value 200; in location Green-37 might be the intensity value 190; and in location Blue-37 might be the value 10 . The visible result would be a rather saturated yellow. Note that a single number, 37 , codes a complex of three intensities that can be varied depending on how the addresses of the lookup table are "loaded."

Therein lies the payoff for using lookup tables, which often seem to involve only needless indirection to novices. Having drawn a complex figure through the use of a great number of individual drawing commands for points, line segments, or arcs using address value 37 , the researcher can instantly alter the color appearance of the entire result (e.g., a face) by changing the contents of just the three color addresses numbered 37 . In fact, a host of imageprocessing operations can be implemented with lookup tables. Of special interest to the study of surface perception are such features as the ability to dynamically change the luminance of complex image regions in real time, the ability to code the lookup table to automatically generate color transparency effects, and the ability to code the table to instantly pick out level curves of intensity (isophotes) in complex shaded images.

Another technological innovation of great use for studying surface perception concerns hidden element removal, which is the suppression of drawing commands for points, 
line segments, or areas of a solid object model because some other opaque element is deemed to occlude it from visibility to the viewer. The $z$ axis in computer graphics is by convention the one along the line of sight to the display screen. It is with reference to this axis that hidden element removal must be performed when rendering images of three-dimensional scenes. One of the classical techniques for performing this task is the maintenance in graphics software of a $z$-buffer, which codes the $z$ coordinates of all currently visible lines and surfaces. The visible entities are those having the lowest $z$ value, given a left-handed coordinate system with $x$ horizontal, $y$ verti$\mathrm{cal}$, and $z$ facing away from the viewer. Thus, before actually sending a command to display a segment on the screen, a software routine checks to see if the $z$ coordinates of that segment are lower than the ones currently in the buffer. If this is not the case, the segment is not displayed. Complications arise if part of the segment is deemed to be visible and other parts are not. Because of the large amount of computer time needed to perform calculations and the programmer expertise and effort required to implement them, hidden element removal remained for a long time the domain of specialists with extensive computational power at their disposal.

Good news is that recently a number of graphics systems affordable to typical perceptual laboratories have become available featuring hardware $z$-buffers. When using such systems, the programmer simply sends threedimensional coordinates and associated intensities to the display processor, which then rapidly checks its hardware $z$-buffer to determine whether the segment is to be visible or suppressed because of occlusion by a nearer, visible segment. Hardware $z$-buffers even perform appropriate interpolations for cases of partial visibility.

More good news for the perceptual psychologist is that increasingly high-level graphics primitives are available in new systems. "Primitive" refers to the display unit referred to in a given graphics command. A "primitive" primitive would be a command to draw a pixel of a given location and intensity. Thus, a command in the gray-level system lacking a lookup table might say "DRAW DOT $(4,3,37)$," where the graphics system understands the first two parameters to refer to screen location and the third to intensity. An example of a high-level primitive might be a command to draw a smoothly shaded, five-sided polygon, having specified $x, y$, and $z$ coordinates for each vertex and having a specified color lookup table address assigned to each vertex. In this case, the color lookup table addresses for visible points on the interior of the polygon would be automatically interpolated from the addresses of the vertex points according to an algorithm internal to the display processor, without further programming by the user or computational load on the host computer. The gains in processing time and programmer ease of use for systems using color lookup tables, hardware $z$-buffers, and high-level primitives over their predecessors are difficult to exaggerate. With such systems, psychologists doing research on surface perception can rapidly move from doing the "same old things" a little more easily to doing new and different things that would not have been attempted a few years ago.

\section{REFERENCES}

Braunstein, M. L. (1976). Depth perception through motion. New York: Academic Press.

FOLEY, J. D., \& VAN DAAM, A. (1984). Fundamentals of interactive computer graphics. Reading, MA: Addison-Wesley.

GiBson, J. J. (1950). The perception of the visual world. Boston: Houghton Mifflin.

MingolLA, E. (1983). Perception of shape and illuminant direction from shading. Unpublished doctoral dissertation, University of Connecticut, Storrs.

Mingolla, E., \& TodD, J. T. (1984). Computational techniques for the graphic simulation of quadric surfaces. Journal of Experimental Psychology: Human Perception \& Performance, 10, 740-745.

Mingolla, E., \& TODD, J. T. (1986). Perception of solid shape from shading. Biological Cybernetics, 53, 137-151.

Newman, W. M., \& Sroull, R. F (1979). Principles of interactive computer graphics (2nd ed.). New York: McGraw-Hill.

PARK, C. S. (1985). Interactive microcomputer graphics. Reading, MA: Addison-Wesley.

TodD, J. T., \& Axerstrom, R. (in press). Perception of threedimensional form from patterns of optical texture. Joumal of Experimental Psychology: Human Perception \& Performance.

TODD, J. T., \& MingollA, E. (1984). Simulation of curved surfaces through patterns of optical texture. Journal of Experimental Psychology: Human Perception \& Performance, 10, 734-739. 